\title{
Finanztransfers im Rahmen der EU-Strukturpolitik
}

Die Europäische Union (EU) stellt seit Jahrzehnten den peripheren Mitgliedstaaten und Regionen erhebliche Finanzmittel im Rahmen der Strukturpolitik zur Verfügung. In der Finanzperiode 2007-2013 sind das gut 348 Mrd. Euro. Und dennoch sind insbesondere die Staaten Südeuropas nicht in ausreichendem Maße wettbewerbsfähig geworden, wie uns die aktuelle Schulden- und Eurokrise drastisch vor Augen führt. Zwar betont Kommissionspräsident Manuel Barroso, künftig die Politik stärker am Wachstum auszurichten (Barroso 2012), doch bleibt die Frage, ob die EU-Strukturpolitik nicht mit erheblichem Mitteileinsatz nur begrenzt Wachstumseffekte erzielt.

Die Strukturpolitik stellt in Bezug auf die Entscheidungs- und Implementationsprozesse vielleicht die innovativste Politik der EU dar. Nicht nur staatliche, sondern auch nicht-staatliche Akteure auf der Ebene der EU, der Mitgliedstaaten und der Regionen sollen gemäß dem Prinzip der Partnerschaft kooperieren. Verordnung 1083 des Rates vom 11. Juli 2006 verlangt die enge Zusammenarbeit zwischen der Kommission und den Mitgliedstaaten und bezieht auch die zuständigen regionalen, lokalen, städtischen und anderen zuständigen Behörden, aber auch die Wirtschaftsund Sozialpartner und die Akteure der Zivilgesellschaft mit ein (Verordnung (EG) Nr. 1083/2006). Die Politikwissenschaft hat diese Formen der Zusammenarbeit unter dem Label „Multi Level Governance“ diskutiert (Marks 1996). Allerdings hat das Partnerschaftsprinzip die Kommission nicht davon abgehalten, die Förderphilosophie der Strukturpolitik maßgeblich zu bestimmen (siehe unten).

Die Strukturpolitik gehört zu den ausgabenintensivsten Politiken der EU. Zwar gab es bei Gründung der Europäischen Wirtschaftsgemeinschaft 1957 schon den Europäischen Sozialfonds (ESF), aber der eigentliche Beginn der Strukturpolitik wird auf 1975 datiert. Damals musste sich diese Politik mit 8,2 Prozent der gesamten EU-Haushaltsmittel bescheiden. 1999 waren es bereits 35,7 Prozent und heute kommt die Strukturpolitik auf 46,8 Prozent. 70,6 Mrd. Euro stehen 2013 an Verpflichtungen zur Verfügung. Keine andere EU-Politik weist eine vergleichbare Dynamik bei den Finanzmitteln auf. Dabei haben die Erweiterungen der EU um strukturschwache Länder (1973 Irland, 1981 Griechenland, 1986 Spanien und Portugal, 2004 acht osteuropäische Transformationsstaaten und 2007 Bulgarien und Rumä- 
nien) eine maßgebliche Rolle gespielt, weil die neuen Mitglieder auf einen Finanzbeistand der EU gedrängt haben.

Statistisch gesehen ist die EU in ihrer Gesamtheit durch die meisten Erweiterungsrunden ,ärmer“ geworden. Betrachtet man die einzelnen Staaten, dann zeigen sich freilich erhebliche Divergenzen. Das wird auch daran deutlich, dass sich das unterschiedliche Entwicklungsniveau im EU-Haushalt widerspiegelt: Nettozahler stehen Nettoempfängern gegenüber. Bei der ersten Gruppe haben von 1999 bis 2008 pro Kopf die Niederländer mit 1.240 Euro den größten Beitrag geleistet, gefolgt von Schweden (995 Euro), Deutschland (852 Euro) und Großbritannien (729 Euro). Bei den Nettoempfängern konnte im selben Zeitraum Griechenland mit 3.945 Euro die größten Zahlungen auf sich verbuchen, gefolgt von Irland (2.948 Euro), Portugal (2.430 Euro) und Spanien (1.369 Euro). Polen, das erst 2004 der EU beigetreten ist und daher zwangsläufig weniger Mittel erhielt, wurde mit 412 Euro begünstigt. ${ }^{1}$

Auch wenn man regional- und sozialpolitische Zielsetzungen der Strukturpolitik unterscheiden kann - die Finanzen für das erste Ziel entstammen dem Europäischen Fonds für Regionale Entwicklung (EFRE), für das zweite Ziel dem Europäischen Sozialfonds (ESF) -, so fällt eine eindeutige Definition für dieses Politikfeld schwer. Pragmatisch kann man formulieren: Strukturpolitik ist das, was aus den europäischen Strukturfonds mit dem wenig präzisen Ziel finanziert wird, den wirtschaftlichen und sozialen Zusammenhalt zu stärken (Axt 2000: 13). Wie in anderen Politikfeldern stellt auch die Strukturpolitik das Ergebnis eines Kompromisses zwischen den Interessen der Mitgliedstaaten und der EU-Organe dar. Strukturschwache Staaten und Regionen wollten sich Rückflüsse aus dem EU-Haushalt sichern. Um diese Einheiten einzubinden und um Integrationsfortschritte abzusichern, waren die finanzstarken Mitgliedstaaten bereit, entsprechende Finanzmittel in den EU-Haushalt einzustellen. Um dabei bloße Mitnahmeeffekte zu unterbinden und eine möglichst effiziente Nutzung der Finanzmittel sicherzustellen, übertrugen die Mitgliedstaaten der Kommission eine wichtige Funktion in der Strukturpolitik. Zum einen spielt sie in der Feinstrukturierung der Politik eine zentrale Rolle und zum anderen interagiert sie in der Programmplanung an herausgehobener Stelle mit den Staaten und Regionen. Wenn hier - wie es in der Wirtschaftswissenschaft formuliert wird - ein Verhältnis von „Prinzipal“ (Mitgliedstaaten) und „Agent“ (Kommission) intendiert war, so hat sich die Kommission als Agent gerade in diesem Politikfeld weitgehend emanzipieren und sich nicht zuletzt wegen des Wissensvorsprungs und der engen Kooperation mit den Regionen einen erheblichen Einfluss auf die Strukturpolitik sichern können.

1 Frankfurter Allgemeine Zeitung, 28.3.2010. 
Wollte man mit der Strukturpolitik weniger entwickelten Mitgliedstaaten eine Unterstützung zukommen lassen, so ging es den entwickelten Staaten aber auch darum, den wenig entwickelten Regionen im eigenen Territorium europäische Finanzhilfen zu sichern. Deutschland konnte auf diese Weise sicherstellen, dass Regionen im Strukturwandel wie zum Beispiel das Ruhrgebiet ebenso begünstigt wurden wie nach 1989 die neuen Bundesländer. Das vertraglich festgehaltene allgemeine Ziel der Strukturpolitik setzt auf den Regionalausgleich. Es heißt in Art. 174 des Vertrags über die Arbeitsweise der EU (AEUV): „Die Union entwickelt und verfolgt weiterhin ihre Politik zur Stärkung ihres wirtschaftlichen, sozialen und territorialen Zusammenhalts, um eine harmonische Entwicklung der Union als Ganzes zu fördern. Die Union setzt sich insbesondere zum Ziel, die Unterschiede im Entwicklungsstand der verschiedenen Regionen und den Rückstand der am stärksten benachteiligten Gebiete zu verringern.“ Art. 175 AEUV ergänzt: „,...] Die Union unterstützt auch diese Bemühungen durch die Politik, die sie mit Hilfe der Strukturfonds (Europäischer Ausrichtungs- und Garantiefonds für die Landwirtschaft Abteilung Ausrichtung, Europäischer Sozialfonds, Europäischer Fonds für regionale Entwicklung), der Europäischen Investitionsbank und der sonstigen vorhandenen Finanzierungsinstrumente führt.“

Der auf den Ausgleich zwischen den Regionen setzende Ansatz der EU-Strukturpolitik lässt sich anschaulich am Beispiel Griechenlands aufzeigen. Griechenland eignet sich deshalb besonders gut, weil das Land seit seinem Beitritt zur EU im Jahr 1981 bis 2008 immerhin 133,5 Mrd. Euro netto durch die EU-Struktur- und Agrarpolitik erhalten hat und weil das Land aktuell im Zentrum der Schuldenkrise steht. Die EU hat Griechenland in der Förderperiode 2007-2013 insgesamt 20,4 Mrd. Euro an Strukturmitteln zur Verfügung gestellt, davon 19,6 Mrd. Euro für den Ausgleich regionaler Disparitäten. Griechenlands nationaler Kofinanzierungsbetrag wurde auf 5,7 Mrd. Euro festgelegt. Im Zuge der Krise wurde dieser Anteil erheblich gesenkt, indem die Kofinanzierungssätze Griechenlands von 20 bis 25 auf 5 Prozent gesenkt wurden. Nahezu 6 Mrd. Euro dienen der Verbesserung der Transport-Infrastruktur, 5,5 Mrd. Euro dem Umweltschutz, mehr als 3,6 Mrd. Euro der Förderung von Forschung und Entwicklung und 2,2 Mrd. Euro der Ausbildungs- und Beschäftigungspolitik (zum Folgenden ausführlicher Axt 2013).

Einige Beispiele mögen veranschaulichen, was in der Förderperiode 2007-2013 gefördert wird: Mit einer Summe von 12.499.600 Euro wird in Griechenland und angrenzenden Regionen das Programm ,Alterenergy for sustainability in the Adriatic" gefördert, das in den kleinen Kommunen des Adriaraumes die Energieeffizienz verbessern soll. In der Bergregion Kozani wird die Verbreitung des „W-Lan-Netzes“ mit 355.000 Euro unterstützt. 1.673.842 Euro steuert die EU für einen Wis- 
senschafts- und Technologiepark in Epirus bei. Mit 2.430.000 Euro finanziert die EU ein Projekt in Griechenland und anderen Staaten, mit dem Städte den Klimawandel bewältigen sollen. Auf 605.235 Euro beläuft sich die EU-Fördersumme, um in der Region Sterea Ellada ein Ausstellungs- und Konferenzzentrum zu errichten. Mit 36.000.000 Euro werden Internetanschlüsse in Hotels über ganz Griechenland finanziert. 50.862.390 Euro kommen von der EU, um das U-Bahn-Netz in Athen zu vervollständigen. Mit 1.200.000 Euro soll das Umweltbewusstsein in Klein- und Mittelbetrieben von Spanien über Italien bis Griechenland gefördert werden. Auf Kreta werden mit 265.000 Euro Bemühungen zu Verwertung wissenschaftlicher Erkenntnisse unterstützt. 37.890.900 Euro wendet die EU auf, um in Thessalien einen ausgetrockneten See wieder ökologisch zu regenerieren, damit die Trinkwasserversorgung verbessert und der regionale Tourismus gefördert wird. 1.310.000 Euro wendet die EU auf, damit auf den Ägäischen Inseln Salzwasser zu Trinkwasser umgewandelt wird. 66.400.000 Euro bringt die EU auf, damit auf der Halbinsel Kassandra in Nordgriechenland eine 17,5 km lange Autostraße gebaut werden kann. 77.308.316 Euro kostet die EU das Egnatia-Straßenprojekt, mit dem von Epirus bis Thrakien der Autoverkehr verbessert werden soll. Und 33.010.000 Euro kommen von der EU, um in Igoumenitsa im Nordwesten Griechenlands den Fährhafen für Verbindungen nach Italien und auf die Ionischen Inseln auszubauen. Für jede dieser Investitionen mag man - für sich genommen - gute Fördergründe geltend machen. Was hier zunächst einmal festzuhalten bleibt, ist der Umstand, dass sich die EUStrukturpolitik bemüht, in den verschiedensten Regionen Griechenlands Maßnahmen zu fördern und das Gefälle zwischen den Regionen abzubauen.

Dass der erhebliche Mitteleinsatz - immerhin hat Griechenland seit $1981 \mathrm{mehr}$ als 50 Milliarden Euro aus den EU-Strukturfonds erhalten -, die ökonomische und finanzpolitische Performanz des Landes nicht nachhaltig verbessert hat, liegt sicher in erheblichem Maße an der im Land weit verbreiteten Praxis von Korruption und Klientelismus. Transparency International platzierte Griechenland 2012 beim Korruptions-Perzeptions-Index auf Platz 94 von 174 Staaten und damit auf den letzten Rang aller EU-Staaten (Transparency International 2012). Aber auch die europäische Strukturpolitik muss sich kritischen Fragen stellen: Hat die bisherige stark auf den Regionalausgleich setzende Strukturpolitik in ausreichendem Maße die Wettbewerbsfähigkeit gefördert, so dass auch strukturschwache Volkswirtschaften an den Vorteilen des Binnenmarktes partizipieren und die Vorteile des Euroraums zur Steigerung ihrer eigenen ökonomischen Leistungsfähigkeit nutzen können? Welche Wachstumseffekte hat die EU-Strukturpolitik bewirkt? Eine zweite Frage schließt sich an: Welche Alternativen zum jetzigen Zuschnitt der EU-Strukturpolitik gibt es und wie sieht es mit der politischen Durchsetzbarkeit aus? 
Wissenschaftler des Mannheimer „Zentrums für Europäische Wirtschaftsforschung" haben die Wirkung der EU-Strukturpolitik am Beispiel von 3.600 Projekten untersucht und sind dabei zu einem ernüchternden Ergebnis gekommen: Lediglich bei 37 Prozent aller untersuchten Projekte konnten Wachstumseffekte ausgemacht werden. ${ }^{2}$,With respect to the share of growth-enhancing spending, we find that the share of spending without growth effects amounts to up to $63 \%$ under the pessimistic scenario." (Zentrum für Europäische Wirtschaftsforschung 2012: 5) Die Wachstumseffekte wurden bei den einzelnen Projekten jeweils danach bewertet, welcher Förderzweck verfolgt wird. So kam man zu der Einschätzung, dass folgende Projekttypen keinen Wachstumseffekt aufweisen: Tourismus und Kultur, urbane Entwicklung, Administration der Strukturfonds, Unternehmensberatung, Verwaltung, soziale Inklusion und territoriale Entwicklungsstrategien. Zumeist moderate Effekte wurden Projekten im Bereich des Gesundheitswesens, der Umwelt und der Energie zugesprochen. Lediglich bei den Bereichen Ausbildung, Transport, Forschung und Entwicklung, Rechtsstaatlichkeit und Kriminalprävention sowie Kommunikation wurden (zumeist) größere Wachstumswirkungen attestiert. Schaut man sich die Liste der von der EU geförderten Projekte in Griechenland an (siehe oben), muss man davon ausgehen, dass in vielen Fällen allenfalls geringe Wachstumseffekte aufgetreten sind.

Schon 1997 hat der Verfasser kritisiert, dass in der EU-Strukturpolitik ein „Gießkannenprinzip“ zur Anwendung komme, weil sich die Förderung nicht auf die primär Bedürftigen konzentriere (Axt 1997: 893). Diese Kritik ist aktuell wieder bestätigt worden: Wenn die EU nicht flächendeckend alle EU-Staaten strukturpolitisch fördern, sondern sich auf die Staaten konzentrieren würde, die am wenigsten entwickelt und die größten Wettbewerbsnachteile haben, könnte sie auch den EUHaushalt erheblich entlasten. Bezogen auf den aktuellen Jahreshaushalt kommen britische Wissenschaftler zu folgendem Ergebnis: „Focusing the EU's structural funds on less wealthy member states and stopping the recycling exercise whereby richer member states subsidise each other's regional development policies would save just over $€ 20$ bn. “(Open Europe 2012: 3) Statt die Förderung zu konzentrieren, begünstigt die EU-Strukturpolitik alle Mitgliedstaaten. Die umfangreichste Förderung kommt dabei aus dem Regionalfonds, so auch in Griechenland.

2 Einbezogen wurden Projekte und Regionen in folgenden EU-Staaten: Frankreich, Deutschland, Italien, Malta, Portugal, Slowakei, Spanien und Großbritannien. Projekte in Griechenland wurden nicht erfasst. Aufgrund der methodischen Anlage der Untersuchung kann man aber die Ergebnisse - wenn auch vielleicht nicht in quantitativer, so doch in qualitativer Hinsicht - durchaus auch auf Griechenland übertragen. 
Dass die EU-Strukturpolitik nur bescheidene Erfolge in der Wachstumsförderung erzielt, ist darauf zurückzuführen, dass bislang der Anspruch maßgeblich war, regionale Disparitäten in und zwischen den EU-Mitgliedstaaten auszugleichen. Dass aber die Förderung von Wachstum und Wettbewerbsfähigkeit als die zentrale Herausforderung gelten muss, hat mittlerweile auch der Europäische Rat eingestanden. Am 21. Juli 2011 hat er betont: "We call for a comprehensive strategy for growth and investment in Greece. We welcome the Commission's decision to create a Task Force which will work with the Greek authorities to target the structural funds on competitiveness and growth, job creation and training. We will mobilise EU funds and institutions such as the EIB [European Investment Bank; Anm. des Autors] towards this goal and relaunch the Greek economy. Member States and the Commission will immediately mobilize all resources necessary in order to provide exceptional technical assistance to help Greece implement its reforms." (Council of the European Union 2011)

Wie können Alternativen zur regionenzentrierten Förderung aussehen? Zwei Kernelemente könnten eine Alternative bieten: Erstens müsste sich die EU vom ehrgeizigen Ziel des Regionalausgleichs verabschieden und die Förderung am Kriterium des nationalen und nicht des regionalen Wohlstands ausrichten (Axt 2005). Das käme einer staatenzentrierten Förderung gleich, was Länder wie Griechenland begünstigen würde, nicht aber wettbewerbsfähige Länder wie beispielsweise Deutschland. Die entwickelten EU-Staaten würden von Zahlungen an die EU entlastet und der verwaltungsmäßige Aufwand in Brüssel würde reduziert. Wenn die entwickelten Staaten die Förderung ihrer schwachen Regionen für notwendig halten, können sie das mit den bei den Überweisungen an die EU eingesparten Finanzmitteln tun. Zweitens müsste statt einer weitgehend flächendeckenden Förderung eine Konzentration auf Wachstumskerne erfolgen. Die Erfahrungen, die man mit der Wirtschaftsförderung Ostdeutschlands gemacht hat, können genutzt werden. Hier hat der "Gesprächskreis Ost" unter Leitung des früheren Hamburger Bürgermeisters Klaus von Dohnanyi unter anderem gefordert, von der flächendeckenden Förderung zur Schwerpunktsetzung überzugehen: „Unerlässlich ist weiterhin eine deutliche Konzentration der Wirtschaftsförderung und Infrastruktur auf regionale Schwerpunkte (Wachstumskerne, Cluster)“ (Dohnanyi/Most o. J.).

Wie sieht es aber mit den Chancen einer durchgreifenden Reform der EU-Strukturpolitik aus? Dass die EU von der Fokussierung auf den Regionalausgleich abgeht, ist wenig wahrscheinlich: Erstens schreibt der zentrale Artikel 174 AEUV die re- 
gionenzentrierte Förderung vor. ${ }^{3}$ Zweitens setzen sich alle bislang begünstigten Regionen für den Erhalt der regionenzentrierten EU-Förderung ein. Drittens ist das Prinzip einer Konzentration der Förderung auf bedürftige Staaten bislang nur von einer Minderheit der EU-Staaten befürwortet worden. ${ }^{4}$ Viertens plädieren auch relativ wohlhabende Nettozahler-Staaten wie Deutschland für den Regionalausgleich, weil ihnen dieser Ansatz wenigstens einen gewissen Rückfluss aus dem EU-Haushalt sichert. Fünftens haben die Befürworter des Regionalausgleichs immer auf den vermeintlich positiven politischen Effekt verwiesen, dass bei einer flächendeckenden Förderung eher europäisches Bewusstsein gefördert werde. Aber selbst in einem so stark von der Strukturpolitik begünstigten Staat wie Griechenland wächst der Euroskeptizismus. Nur noch 11 Prozent der im Jahr 2013 Befragten gaben an, dass die europäische Integration die Wirtschaft gestärkt hat. 2012 waren es noch 18 Prozent. Und lediglich 33 Prozent äußerten sich positiv über die EU; im Vorjahr waren es noch 37 Prozent (Pew Research Center 2013: 1).

\section{Literatur}

Axt, Heinz-Jürgen, 1997: Strukturpolitik und Kohäsion in der Europäischen Union: Reform in der Perspektive der Osterweiterung, in: Zeitschrift für Politikwissenschaft 7 (3), 885-927.

Axt, Heinz-Jürgen, 2000: EU-Strukturpolitik. Einführung in die Politik des wirtschaftlichen und sozialen Zusammenhalts, Opladen.

Axt, Heinz-Jürgen, 2005: Alter Wein in neuen Schläuchen. Warum die Kommission nicht vom Ziel des Regionalausgleichs in der Strukturpolitik abgeht, in: Ines Hartwig/Wolfgang Petzold (Hrsg.), Solidarität und Beitragsgerechtigkeit. Die Reform der EU-Strukturfonds und die Finanzielle Vorausschau, Baden-Baden, 11-37.

Axt, Heinz-Jürgen, 2013: Griechenland: trotz europäischer Fördergelder nicht wettbewerbsfähig, in: Südosteuropa-Mitteilungen 53 (2), 6-24.

Barroso, José Manuel, 2012: Rede von Präsident Barroso zum mehrjährigen Finanzrahmen 2014-2020 bei der Debatte des Europäischen Parlaments vor der Tagung des Europäischen Rates, 21.11.2012, Straßburg, http://europa.eu/rapid/ press-release_SPEECH-12-848_de.htm (Stand 22.5.2013).

3 Deshalb ist auch nicht zu erwarten, dass bei der aktuell zu beschließenden Mittelfristigen Finanzperiode 2014-2020 eine grundlegende Veränderung der Strukturpolitik erfolgt (Axt 2013).

4 Als es um die Beschlussfassung zur Finanzperiode 2007 bis 2013 ging, machten sich lediglich Großbritannien, Schweden und die Niederlande für ein Abgehen vom Ziel des Regionalausgleichs stark (Axt 2005: 19-21). 
Council of the European Union, 2011: Statements by the Heads of State or Government of the Euro Area and Institutions, 21.7.2011, Brussels, http://www.con silium.europa.eu/uedocs/cms_data/docs/pressdata/en/ec/123978.pdf (Stand: 22.5.2013).

Dohnanyi, Klaus von/Most, Edgar, o. J.: Für eine Kurskorrektur beim Aufbau Ost, http://www.fifoost.org/allgemein/divers/aufbauost/node2.php

(Stand: 22.5.2013).

Marks, Gary, 1996: Exploring and Explaining Variation in EU Cohesion policy, in: Liesbet Hooghe (Hrsg.), Cohesion Policy and European Integration: Building multi-Level-Governance, Oxford, 387-422.

Open Europe, 2012: Seizing the Moment: Aligning the EU budget with Europe's economic needs, http://www.openeurope.org.uk/Content/Documents/Pdfs/ 2012EUbudget.pdf (Stand: 22.5.2013).

Pew Research Center, 2013: The New Sick Man of Europe: The European Union, http://www.pewglobal.org/2013/05/13/the-new-sick-man-of-europe-the-europ ean-union (Stand: 22.5.2013).

Rat der Europäischen Union, 2006: Verordnung (EG) Nr. 1083/2006 vom 11. Juli 2006 mit allgemeinen Bestimmungen über den Europäischen Fonds für regionale Entwicklung, den Europäischen Sozialfonds und den Kohäsionsfonds und zur Aufhebung der Verordnung (EG) Nr. 1260/1999, http://eur-lex.europa. eu/LexUriServ/LexUriServ.do?uri=OJ:L:2006:210:0025:0078:DE:PDF, (Stand: 20.5.2013).

Transparency International, 2012: Corruption Perceptions Index 2012, http:// cpi.transparency.org/cpi2012/results (Stand: 22.5.2013).

Zentrum für Europäische Wirtschaftsforschung, 2012: Growth Enhancing Expenditure in EU Cohesion Spending from 2007 to 2013, Mannheim, http://ftp.zew. de/pub/zew-docs/gutachten/ZEW_Cohesion2012.pdf (Stand: 22.5.2013).

Korrespondenzanschrift:

Prof. em. Dr. Heinz-Jürgen Axt

Institut für Politikwissenschaft

Universität Duisburg-Essen

Lotharstraße 65

47057 Duisburg

E-Mail: heinz-juergen.axt@uni-due.de 